PAKES AND JOLLYMAN : COLLECTION AND EXAMINATION

\title{
XXXIII.-The Collection and Examination of the Gases produced by Bacteria from certain Media.
}

By Walter Charles Cross Pakes and Walter Henry Jolly an.

DURING the course of an investigation upon the bacterial flora of the water of a certain well, several specimens of bacteria were isolated which belonged to the group of Bacillus fuorescens liquefaciens. The determination of the cultural reactions enabled us to divide these into two groups, (1) that which produced gas, and (2) that which produced no gas in media containing nitrate. These were provisionally designated S.O.7 and S.O.6 respectively. In order to obtain more information concerning the former of these, it was decided to analyse the gas produced.

As the various forms of apparatus hitherto described for the purpose of the collection of gas thus produced did not seem to be sufficiently accurate or suitable for our purpose, we designed one which is both simple and accurate.

It was necessary to have an apparatus which fulfilled the following conditions :

(1) A relatively large amount of medium must be used-from 300 to 500 c.c.

(2) The gas receiver must have a capacity of at least 600 c.c.

(3) It must be easy to inoculate the medium without any chance of accidental contamination.

(4) The receiver must be fitted with taps so that a part or the whole of the gas can be removed during the course of the experiment without chance of contamination.

(5) Every part must be capable of effective sterilisation both before inoculation and at the conclusion of the experiment.

In our apparatus, the receiver is a bell-jar made of stout glass about $14 \mathrm{~cm}$. high and $9 \mathrm{~cm}$. in diameter. At the apex a glass tube is fused in ; this rises vertically $5 \mathrm{~cm}$, and is then bent at right angles, the arm being about $10 \mathrm{~cm}$. long. Halfway along the vertical tube is a three-way tap placed horizontally. This receiver is placed in an iron cylinder three parts full of mercury, about 35 pounds of mercury being required for each apparatus. The flask containing the medium is an ordinary one of from 300 to 1500 c.c. capacity, and in this are placed from 100 to 400 c.c. of the medium. The mouth is fitted with a rubber stopper perforated in the centre, and carrying a tube bent at right angles; this carries a piece of pressure tubing about three inches long, which is plugged with a small piece of cotton-wool at the open end.

When the apparatus is to be used, the mercury in the receiver is 
sucked up into the tap, which is then shut off. The open end of the three-way tap is plugged with melted paraffin wax, and the empty portion of the glass tube filled with sterile water. The inoculation is made in the usual way with a platinum needle, care being taken that the neck of the flask and the rubber stopper are thoroughly heated in a flame before and after the operation. The cotton-wool plug is removed from the end of the rubber tubing, and the free end of the latter is slipped on to the arm of the receiver. All the connections, including the three-way tap after it has been turned so as to connect the flask with the receiver, are carefully painted with melted paraffin wax. When the tap is turned, the mercury naturally falls in the receiver, and the

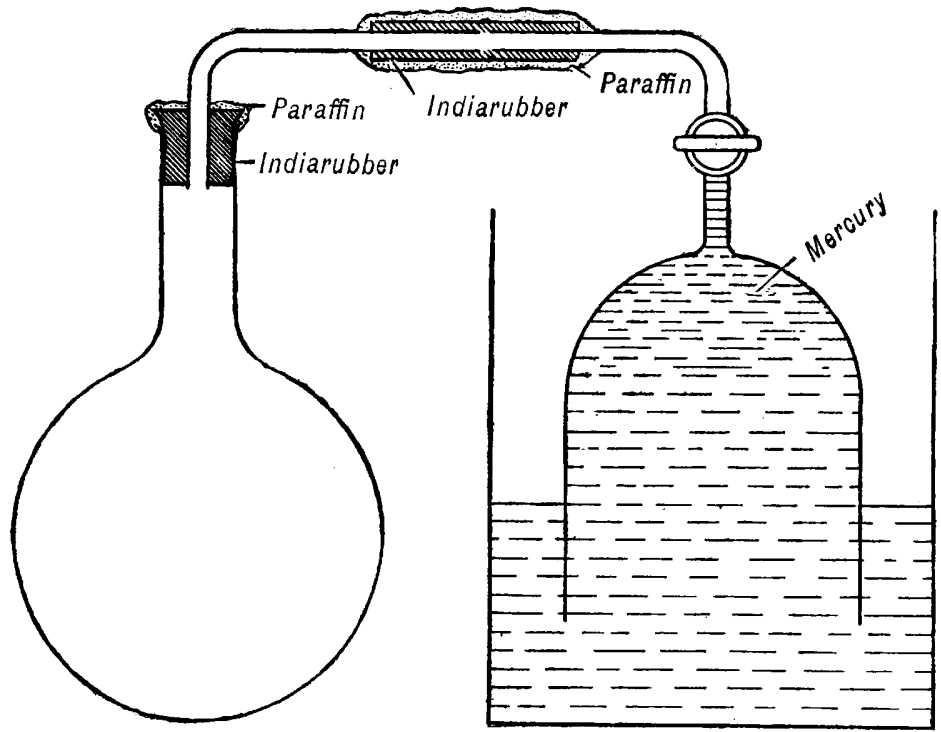

height of the mercury may be marked when the temperature of the whole apparatus is that of the incubator.

In order to test the apparatus, we performed several blank experiments. We filled the flask with water, connected it with the receiver, and then withdrew a certain quantity of mercury from the reservoir so that the difference between the levels of the mercury was between four and five inches. We then allowed the apparatus to stand in the laboratory for a week, and examined it daily. At the end of the time there was absolutely no fall in the mercury. On nine occasions, we have inoculated media for the purpose of determining whether any gas was produced, and although the growth has been allowed to continue for upwards of a week-in one case three weeks-there was no trace of 
gas in the receiver. These trial experiments, taken in conjunction with the fact (p. 329) that no oxygen was found in the receiver in the experiments with the $B$. coli communis in sugar-containing media, show that the apparatus is air-tight, and that there is no chance of air getting into the receiver during an experiment. It is possible that a certain amount of carbon dioxide may be absorbed by the indiarubber, but as the two glass tubes which are joined by the rubber tubing are in actual contact, the amount absorbed by this must be infinitesimal. A certain small amount may be absorbed by the indiarubber stopper, but we do not see how this can be avoided.

In order to examine the gas produced, as it may be inaccurate to take a sample from the receiver, it is necessary to mix the gas in the flask with that in the receiver. This may be rapidly accomplished in the following way.

Connect an Orsat, or some similar gas apparatus (working with mercury) to the three-way tap, remove about 100 c.c. from the receiver, and then, reversing the three-way tap, force the gas into the flask. Now remove about 100 c.c. of the mixed gases from the flask and replace in the receiver. Repeat this three or four times and the gas will be homogeneous throughout. If it is required to have an atmosphere containing an excess of oxygen, a measured volume may be introduced into the receiver from a gas analysis apparatus, and may be mixed in the manner above detailed.

If it is desired to experiment with an atmosphere of pure oxygen, the stopper is provided with a second glass tube through which oxygen is passed after the fluid has been inoculated, care being taken that it is previously filtered through cotton-wool. The tube is finally sealed off.

In order to be prepared to examine small quantities of gas, we used a special Orsat apparatus provided with two gas tubes of extra length and graduated throughout, containing 120 and 60 c.c. respectively, both being water-jacketed.

Experiment No. 1.-Two hundred c.c. of meat extract (that is, the extract of $1 \mathrm{lb}$. of lean meat in 1 litre of water), containing 1 per cent. of ammonium nitrate and 0.5 per cent. of common salt, were placed in a flask of 350 c.c. capacity. After having been sterilised, it was inoculated with a trace of an agar culture of the S.0.7.

The apparatus was placed in the incubator at $20^{\circ}$, and after 2 hours the level of the mercury was marked. On the following day there was no sign of gas in the flask, although there was an abundant growth, but the level of the mercury in the gas jar had risen about half an inch. This decrease in the volume of the gas is due to the fact that the oxygen is taken up by the bacteria in the first stage of the experiment.

On the succeeding day, there was an abundant evolution of gas, and 
the level of the mercury was lower than at the commencement of the experiment.

At the end of four days, the evolution of gas had ceased, and there were 150 c.c. in the receiver. This was analysed, and gave the following result :

$\begin{array}{lcc}\text { Carbon dioxide................ } & 9 \cdot 3 \text { per cent. } \\ \text { Oxygen ......................... } & 0 \cdot 6 \quad " \\ \text { Nitrogen } \ldots \ldots \ldots \ldots \ldots \ldots \ldots \ldots . . . \ldots & 90 \cdot 1 \quad "\end{array}$

Since the original 150 c.c. of air contained approximately 120 c.c. of nitrogen and 30 c.c. of oxygen, there was
A loss of oxygen
amounting to
28 c.c.
A gain of nitrogen
150,
A ", of carbon dioxide,
28,

A second experiment with the same medium and under the same conditions gave
A loss of oxygen
amounting to
33 c.c.
A gain of nitrogen
112 "
A ", of carbon dioxide ",
21,

The facts that although a considerable amount of the oxygen appeared to have been used up before there was any production of gas, and that the organism was still able to grow, suggested that bacteria might be able to obtain oxygen from nitrates. This suggestion was borne out when we found that exactly the same thing happened with the $B$. pyocyaneus grown upon this medium.

In order to test this theory, the B. pyocyaneus was grown in nitrate broth under strictly anaerobic conditions, both in Buchner's tubes and in an atmosphere of hydrogen. In every case, we found that the organism grew, so far as we could see, as well as under aerobic conditions.

As the B. pyocyaneus is generally considered a strictlyaerobic organism (Lehmann and Neumann say that this bacillus will not grow anaerobically, and Flugge says, "Der Pyocyaneus ist ein fast obligater Aerobier"), it is obvious either that it is not a strict aerobe, or that the term anaerobe requires modification.

Our first experiments were conducted with ammonium nitrate, and thinking that the effect was possibly due to the ammonium, we next substituted potassium, sodium, lithium, and calcium nitrates, with identical results.

If the presence of nitrate in a medium renders the medium aerobic, a strict anaerobe ought not to grow in it. Broth and glucose-formate broth therefore were made, and to some of each 1 per cent. of nitrate was added. The bacilli of malignant ndema and of symptomatic 
anthrax were inoculated into these, and incubated anaerobically for several days at $37^{\circ}$. In each of the broth and glucose-formate broth tubes, abundant growth had taken place, but in none of the tubes containing nitrate was there a semblance of growth.

It may be suggested that the nitrate really acts as a poison to these bacteria, but if this is so, it seems strange that so many bacteria, including many which grow either aerobically or anaerobically, grow as well in broth containing nitrate as in simple broth, and that some, such as $B$. anthracis and Streptococcus often grow better.

The term anaerobe must therefore be extended, and it must be understood that an anaerobic organism is one which will not grow in the presence either of free oxygen or of available oxygen in the form of nitrates. The meaning of the term aerobe must be similarly extended.

The gas produced by the $B$. pyocyaneus when grown under anaerobic conditions was next examined. Instead of using some inert gas, such as nitrogen in lieu of air, we determined to eliminate all gas from the apparatus. In order to accomplish this, the following method was adopted.

The medium, about 450 c.c., is placed while very hot in a strong round-bottomed Jena flask, which is filled to the top and is fitted with a good indiarubber stopper, through which passes a piece of manometer tubing bent at right angles; each arm is about $5 \mathrm{~cm}$. in length. The free end of this is connected by means of pressure tubing with a second piece of tubing also bent at right angles, the short arm being about $5 \mathrm{~cm}$, and a longer arm about $20 \mathrm{~cm}$. in length. The longer arm passes through a plug of cotton wool into an Erlenmeyer flask of about 150 c.c. capacity containing 100 c.c. of the medium. The two flasks thus connected together are sterilised in the usual way. When the sterilising is completed, the flasks are removed at once from the steriliser and the tubing connecting them is securely clamped. As the fluid cools, it contracts, leaving an empty space in the neck of the flask. When the fluid has cooled sufficiently to be inoculated, a test-tube containing a young and vigorously growing culture of the Bacillus in the same nitrate medium is taken, the arm of the bent tube which is in the Erlenmeyer flask is withdrawn from the flask and inserted into the test-tube ; the clamp on the rubber tubing is slightly released and a little of the culture is allowed to enter the flask. When five or six c.c. have thus been admitted, the rubber tube is again clamped and the glass tube inserted into a flask containing some recently boiled, quickly-cooled distilled water; the clamp is again released and the inoculated flask is filled entirely; the clamp is secured, and the long. armed glass tubing removed. The horizontal arm of the receiver and the free end of the rubber tubing are both tilled with hot water, and 
the rubber tubing is pushed on to the arm of the receiver (see fig., p. 323). In order to prevent the mercury being sucked back into the flask, care should be taken that it is warmed, and that the medium is thoroughly cooled.

Experiment No. 3.-A flask containing 425 c.c. of meat extract, with 1 per cent. of ammonium nitrate and 0.5 per cent. of common salt, was inoculated with the $B$. pyocyaneus and incubated at $37^{\circ}$.

After four days, when there was no further sign of gas production, the gas was removed 100 c.c. at a time and analysed. The total amount of the gas at the temperature of the laboratory was $469 \cdot 3$ c.c., which was found to contain :

$\begin{array}{rll}59 \cdot 6 & \text { c.c. of } & \text { carbon dioxide. } \\ 2 \cdot 5 & \Rightarrow & \text { oxygen. } \\ 407 \cdot 2 & \Rightarrow & \text { nitrogen. }\end{array}$

The medium was very alkaline after the experiment.

Neither the carbon dioxide nor the oxygen was anticipated, and further experiments were therefore undertaken to ensure the correctness of the result.

Grimbert (Ann. Inst. Pasteur, 1899, 13, 67) found that the $B$. pyocyaneus when grown in media containing nitrate gave a theoretical yield of nitrogen from the nitrate, but that no carbon dioxide was produced. He says that all this gas is combined with the base of the nitrate to form the corresponding carbonate.

This experiment differed in three respects from those of Grimbert, first he used peptone solution instead of broth containing meat extract, secondly he employed potassium nitrate and we employed ammonium nitrate, and thirdly, his experiment was begun under aerobic conditions. The experiments were therefore repeated under conditions as similar to his as possible.

Experiment No. 4.-Four hundred and twenty-five c.c. of meat juice containing 1 per cent. of Witte's peptone, 1 per cent. of ammonium nitrate, and 0.5 per cent. of common salt, inoculated with the $B$. pyocyaneus and grown anaerobically produced, after four days, 268 c.c. of gas having the following composition :

Carbon dioxide, 10.9 ; oxygen, 0.3 ; nitrogen, 88.6 per cent.

A second experiment (No. 5) with the same medium gave gas containing

Carbon dioxide, $11 \cdot 1$; nitrogen, 88.4 per cent.

Next, potassium nitrate was substituted for ammonium nitrate.

Experiment No. 6.-One hundred and fifty c.c. of meat extract containing 1 per cent. of potassium nitrate and 0.5 per cent. of common salt were placed in a flask of 360 c.c. capacity, inoculated with $B$. 
pyocyaneus and grown aerobically. The analysis of the gas at the end of the experiment showed :
A loss of oxygen amounting to $40 \cdot 6$ c.c.
A gain of nitrogen , 114.7,
A , of carbon dioxide ", $28 \cdot 6$,

Experiment No. 7.-Three hundred c.c. of the same medium were placed in a flask of 750 c.c. capacity. The analysis showed :
A loss of oxygen amounting to 68.7 c.c.
A gain of nitrogen " 264.6,
A " of carbon dioxide , 46.7 ,

The same medium inoculated with the B. pyocyaneus and grown anaerobically produced gas containing

Carbon dioxide, 23 ; oxygen, $1 \cdot 7$; nitrogen, 75 per cent.

A further experiment on exactly similar lines gave gas with the following composition:

Carbon dioxide, 12.8 ; oxygen, 0.7 ; nitrogen, 86.4 per cent.

To the medium used in the last two experiments 1 per cent. of Witte's peptone was added, and the gas produced was found to contain :

Carbon dioxide, 10.2 ; oxygen, 0.6 ; nitrogen, 89.2 per cent.

Grimbert's results were obtained with Cola's peptone. We were unfortunately unable to obtain any of this at the time, but we tried experiments with Chapoteau's peptone.

Experiment No. 11.-Four hundred and ninety c.c. of distilled water containing 1 per cent. of potassium nitrate, 1 per cent. of peptone, and 0.5 per cent. of common salt, were inoculated with the organism and in the resulting gas there was 4.5 per cent. of carbon dioxide. The growth on this occasion was not so abundant as usual, probably owing to the use of distilled water.

As Grimbert did not analyse the gas obtained in his experiments until the organism had been growing for 34 days, we analysed the gas produced at the end of each day.

Experiment No. 12.-Four hundred and sixty-five c.c. of meat extract containing 1 per cent. of peptone, 1 per cent. of potassium nitrate, and 0.5 per cent. of common salt were inoculated and incubated anaerobically on the 10th of the month.

On the 14th, 156 c.c. of gas were found of the composition:

Carbon dioxide, 13.4 ; oxygen, 0.4 ; nitrogen, 86.2 per cent.

On the 15 th, $104 \cdot 8$ c.c. of gas containing :

Carbon dioxide, 11.0 ; oxygen, 0.4 ; nitrogen, $88 \cdot 6$ per cent. 
On the 16th, 104 c.c. of gas containing :

Carbon dioxide, 116 ; oxygen, 0.4 ; nitrogen, 88.0 per cent.

On this day, all the gas was not removed, about 30 c.c. being left.

On the 20th, all evolution of gas appeared to have ceased; 59 c.c. of gas were collected and were found to contain :

Carbon dioxide, $7 \cdot 6$; oxygen, $0 \cdot 4$; nitrogen, 92.0 per cent.

Although the apparatus was left for a further two days, there was no more gas, and the medium was tested for alkalinity, which was found to be equal to 120 c.c. of decinormal soda per 100 c.c. There was no reaction for nitrites.

We cannot but conclude, therefore, that Grimbert was wrong in stating that the $B$. pyocyaneus does not produce carbon dioxide from a medium containing nitrate.

The production of free oxygen and free nitrogen at the same time suggests a reaction so peculiar that we do not propose to endeavour to explain it, but to content ourselves with calling attention to the fact. There can, we think, be no doubt as to the production of the oxygen because the above analyses were carried out during the time that analyses of the gases produced by the $B$. coli communis from grape sugar were being made, and in no single case did we find any difference in the reading after the gas had been passed over the alkaline pyrogallol.

Further experiments upon this subject are being undertaken and will be published in a subsequent paper. 